\title{
cAMP signaling increases histone deacetylase 8 expression via the Epac2-Rap1A-Akt pathway in H1299 lung cancer cells
}

\author{
Ji-Yeon Park ${ }^{1,2}$ and Yong-Sung Juhnn ${ }^{1,2}$
}

This study was performed to investigate the signaling pathway that mediates cyclic AMP (cAMP)-induced inhibition of histone deacetylase 8 (HDAC8) degradation, and the effect and underlying mechanisms of the resulting increase in HDAC8 expression on cisplatin-induced apoptosis in lung cancer cells. CAMP signaling increased HDAC8 expression via a protein kinase A (PKA)independent pathway in $\mathrm{H} 1299$ non-small cell lung cancer cells. However, treatment with a selective activator of an exchange protein that was activated by CAMP (Epac) increased HDAC8 expression, and Epac2 inhibition abolished the isoproterenol (ISO)-induced increase in HDAC8 expression. ISO and the Epac activator activated Rap1, and Rap1A activation increased HDAC8 expression; moreover, inhibition of Rap1A with a dominant negative Rap1A or by shRNA-mediated knockdown abolished the ISO-induced increase in HDAC8 expression. Activation of cAMP signaling and Rap1A decreased the activating phosphorylation of Akt. Akt inhibition with a pharmacological inhibitor or expression of a dominant negative Akt inhibited the MKK4/JNK pathway and increased HDAC8 expression. The Akt inhibitor-induced increase in HDAC8 expression was abolished by pretreatment with proteasomal or lysosomal inhibitors. The ISO treatment increased cisplatin-induced apoptosis, which was abolished by HDAC8 knockdown. Exogenous HDAC8 expression increased cisplatin-induced apoptosis and decreased TIPRL expression, and the knockdown of TIPRL increased the apoptosis of cisplatin-treated cells. The ISO treatment decreased cisplatin-induced transcription of the TIPRL gene in a HDAC8-dependent manner. In conclusion, the Epac-Rap1-Akt pathway mediates CAMP signaling-induced inhibition of JNK-dependent HDAC8 degradation, and the resulting HDAC8 increase augments cisplatin-induced apoptosis by repressing TIPRL expression in H1299 lung cancer cells.

Experimental \& Molecular Medicine (2017) 49, e297; doi:10.1038/emm.2016.152; published online 24 February 2017

\section{INTRODUCTION}

The cyclic AMP (cAMP) signaling pathway is activated by cAMP, which is formed by adenylate cyclases and degraded by phosphodiesterases. Adenylate cyclases are activated by the stimulatory $G$ protein, which is activated by receptors for a variety of signals, including epinephrine and prostaglandin E2. The increase in cAMP levels activates target molecules, such as cAMP-dependent protein kinase (protein kinase A, PKA), exchange protein directly activated by cAMP (Epac) and cyclic nucleotide-gated ion channels. ${ }^{1}$ These target effector molecules regulate various cellular responses, including metabolism, gene expression, proliferation and apoptosis. Therefore, cAMP signaling has been studied as a target for various disease treatments, including cancer. ${ }^{2}$ Various alterations to key molecules of the cAMP signaling pathway have been observed in lung cancer, and phosphodiesterase inhibitors have been shown to synergize with cisplatin to induce apoptosis in a broad panel of human lung cancer cell lines. These findings present cAMP signaling as a promising cellular target for antitumor treatments. ${ }^{3,4}$

Histone deacetylases (HDACs) are enzymes that catalyze the removal of acetyl groups from lysine residues in histones and non-histone proteins to regulate gene transcription and numerous other biological processes, such as cell proliferation, differentiation and human cancer development. ${ }^{5,6}$ Histone deacetylase 8 (HDAC8) is a class I HDAC and is composed of 377 amino acids that are encoded by a gene on chromosome $\mathrm{Xq} 21.2-\mathrm{Xq} 21.3 .^{7}$ HDAC8 mRNA expression is observed in various human tissues and tumor cell lines, and the HDAC8 protein is found in both the cytosol and the nucleus. HDAC8 deacetylates histones and non-histone proteins, such as heat-shock protein 20 and estrogen-related receptor $\alpha{ }^{8,9}$ and

\footnotetext{
${ }^{1}$ Department of Biochemistry and Molecular Biology, Seoul National University College of Medicine, Seoul, Korea and ${ }^{2}$ Department of Biomedical Sciences and Cancer Research Institute, Seoul National University College of Medicine, Seoul, Korea

Correspondence: Professor Y-S Juhnn, Department of Biochemistry and Molecular Biology, Seoul National University College of Medicine, 103 Daehak-ro, Jongno-gu, Seoul 03080, Korea.

E-mail: juhnn@snu.ac.kr

Received 11 October 2016; accepted 13 October 2016
} 
HDAC8 has important roles in transcription, proliferation, invasion and apoptosis in several tumor types, including lung cancer. Thus, HDAC8 is a challenging and promising target for pharmacological intervention. ${ }^{10}$

cAMP signaling regulates protein levels by controlling gene transcription via transcriptional activators, (that is, the cAMP response element binding (CREB) protein) and by controlling protein degradation via the proteasome and autophagy. ${ }^{11,12}$ Recently, we found that cAMP signaling increases HDAC8 expression by inhibiting its JNK-dependent degradation via autophagy and the proteasome system in lung cancer cells. ${ }^{13}$ However, the signaling pathway that mediates the inhibition of the HDAC8 degradation by cAMP is unclear, and the physiological significance of the cAMP-induced increase in HDAC8 expression remains unelucidated. We have investigated the role of cAMP signaling in lung cancer cells by triggering apoptosis with ionizing radiation and anticancer drugs. An HDAC8-specific inhibitor reportedly induces apoptosis in T-cell lymphomas. ${ }^{14}$ Therefore, this study was performed to investigate the signaling pathway that mediates cAMP-induced inhibition of HDAC8 degradation to increase its expression. We also investigated the effect of the HDAC8 expression increase on cisplatin-induced apoptosis and the underlying mechanisms in lung cancer cells.

\section{MATERIALS AND METHODS}

\section{Cell culture and reagents}

Human non-small cell lung cancer cells (H1299 and A549) were purchased from the Korean Cell Line Bank (KCLB, Seoul, Korea). H1299 and A549 cells were maintained in Dulbecco's Modified Eagle's Medium (DMEM) and RPMI 1640, respectively, in a $\mathrm{CO}_{2}$ incubator at $37^{\circ} \mathrm{C}$; the media were supplemented with $10 \%$ fetal bovine serum (Welgene, Gyeongsan, Korea) and $100 \mathrm{U} \mathrm{ml}^{-1}$ penicillin/streptomycin (Welgene). Isoproterenol (ISO), H-89, prostaglandin E2, cycloheximide, cisplatin, lithium chloride, ammonium chloride, chloroquine and dimethylsulfoxide (DMSO) were purchased from Sigma-Aldrich (St Louis, MO, USA). LY294002, wortmannin and MG-132 were purchased from Calbiochem (Nottingham, UK), and 8-pCPT-2'-OMe-cAMP and ESI-05 were obtained from the Biological Life Science Institute (Bremen, Germany). Lipofectamine 2000 was purchased from Invitrogen (Carlsbad, CA, USA). PCI-34051 was purchased from Selleck Chemicals (Houston, TX, USA) and NCC-149 was purchased from Tokyo Chemical Industry (Tokyo, Japan).

\section{Expression plasmids and transient transfection}

A constitutively active EE-tagged mutant, Gos (GosQ227L), in the pcDNA3.1+ vector was purchased from Missouri S\&T cDNA Resource Center (Rolla, MO, USA). A dominant negative mutant of PKA (dnPKA) was a gift from G. Stanley McKnight (University of Washington, WA, USA). The green fluorescent protein (GFP)-tagged PKA catalytic subunits (GPKA) were provided by Steven H Green (University of Iowa, IA, USA). The pRK5 expression plasmids that encoded constitutively active Rap1A-V12 (caRap1A) and dominant negative Rap1A-N17 (dnRap1A) were kind gifts from J O'Neill (Trinity College, Ireland). Wild-type HDAC8 was provided by Jaeku Kang (Konyang University, Daejeon, Korea). A dominant negative mutant form of PI3K $(\Delta \mathrm{p} 85)$ was obtained from Dr Julian Downward (The Francis Crick Institute, London, UK). A dominant negative mutant of Akt1 in pUSEamp (dnAktl) was provided by ByungLan Lee (Seoul National University College of Medicine, Seoul, Korea). The plasmids that expressed short hairpin RNA sequences against HDAC8, Epac1, Epac2 and Rap1A were purchased together with the control scrambled shRNA from Sigma-Aldrich. Small interfering RNAs (siRNAs) against HDAC8 and Tip41 (TIPRL) and the control siRNA were purchased from Santa Cruz Biotechnology (Dallas, TX, USA). The plasmids were transfected using Lipofectamine 2000 (Invitrogen) according to the manufacturer's instructions.

\section{Western blot analysis}

Western blotting was performed as previously described. ${ }^{15}$ The following primary antibodies were used: antibodies against HDAC8,

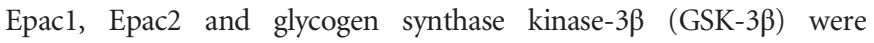
purchased from Santa Cruz Biotechnology; antibodies against cAMP response element binding protein (CREB), $\mathrm{p}-\mathrm{CREB}, \mathrm{p}-\mathrm{PKA}$ substrates, Rap1, p-Akt, Akt, p-GSK-3 $\beta$, p- $\beta$-catenin, p-MKK4, MMK4, p-JNK, JNK, Myc, poly (ADP-ribose) polymerase (PARP), cleaved caspase-3 and cleaved caspase- 9 were purchased from Cell Signaling Technology (Beverly, MA, USA); antibodies against TIPRL were purchased from Abcam (Cambridge, UK); an antibody against $\beta$-actin was purchased from Sigma-Aldrich. The proteins were visualized with the Enhanced Chemiluminescence (ECL) reagent (Thermo Fisher Scientific, Waltham, MA, USA) and detected using an LAS-3000 luminescent image analyzer (Fuji, Tokyo, Japan). The densities of the visualized bands were quantified using the Multi Gauge v.2.3 software (Fuji). The densities were normalized to corresponding control densities and expressed relative to the control.

Quantitative reverse transcription polymerase chain reaction Quantitative polymerase chain reaction ( $\mathrm{qPCR}$ ) was performed in a $20 \mu \mathrm{l}$ mixture that consisted of $250 \mathrm{~nm}$ forward and reverse primers and SYBR Ex Taq (Takara Shuzo, Otsu, Japan) in a C1000 thermal cycler (Bio-Rad, Hercules, CA, USA). The PCR parameters were as follows: $30 \mathrm{~s}$ at $95^{\circ} \mathrm{C}$ followed by 40 cycles of $95^{\circ} \mathrm{C}$ for $5 \mathrm{~s}$ and $55^{\circ} \mathrm{C}$ for $31 \mathrm{~s}$. After 40 cycles, the average threshold cycle (Ct) values from triplicate PCR reactions were normalized against the average $\mathrm{Ct}$ values of GAPDH. The qPCR primer sequences were as follows: HDAC8, forward primer $5^{\prime}$-ATCTCAATGATGCTGTCCTGG-3', reverse primer 5'-CATGATCTGGGATCTCAGAGG-3'; TIPRL, forward primer 5'-GCTGTTGCGGTTTTTCTTGA-3', reverse primer 5'-AGAGGGAA GGTGGAACATGC-3'; GAPDH, forward primer 5'-ACCACAGTC CATGCCATCAC-3', reverse primer $5^{\prime}$-TCCACCACCCTGTTGC TGTA-3'.

\section{Rap1 activation assay}

pGEX Ral GDS-RA, a plasmid for the expression of GST-RalGDSRBD, was provided by Dr Jay $\mathrm{H}$ Chung (National Institutes of Health, Bethesda, MD, USA). The plasmid was transformed into Escherichia coli (Rosetta strain), and protein expression was induced by adding isopropyl $\beta$-D-thiogalactopyranoside (IPTG) to the culture. The bacterial lysate ( $50 \mu \mathrm{l}$ per sample) that contained the GST-RalGDSRBD fusion protein was mixed with glutathione-Sepharose $4 \mathrm{~B}$ beads (GE Healthcare Life Sciences, Pittsburgh, PA, USA) for $1 \mathrm{~h}$ at $4{ }^{\circ} \mathrm{C}$ on a rotator. After the beads were washed three times with the lysis buffer (50 mм Tris-HCl, pH 7.5, $150 \mathrm{~mm} \mathrm{NaCl}, 5 \mathrm{~mm} \mathrm{MgCl}, 1 \%$ Nonidet P-40, 5\% Glycerol, $1 \mathrm{~mm} \mathrm{Na} \mathrm{VO}_{4}, 5 \mathrm{~mm} \mathrm{NaF}, 1 \mathrm{~mm}$ PMSF and a protease inhibitor mixture), $500 \mu \mathrm{g}$ of the cell lysate was added to and mixed with the beads for $1 \mathrm{~h}$ at $4{ }^{\circ} \mathrm{C}$ on a rotator. The beads were washed three times with the lysis buffer. The bound proteins were 
eluted with $2 \times$ loading buffer and boiled for $5 \mathrm{~min}$ at $100^{\circ} \mathrm{C}$. The eluted proteins were analyzed by western blot.

\section{Flow cytometry}

H1299 cells were treated with cisplatin $(50 \mu \mathrm{M})$ and incubated for $48 \mathrm{~h}$. The cells were then washed with phosphate-buffered saline and harvested by trypsinization and centrifugation at $500 \mathrm{~g}$ for $3 \mathrm{~min}$. The cells were incubated in an annexin V buffer that contained FITCannexin $\mathrm{V}$ and propidium iodide (PI) for $15 \mathrm{~min}$ in the dark. Ten thousand fluorescent cells per sample were detected using the FACSCanto II flow cytometer (BD Biosciences, Franklin Lakes, NJ, USA).

\section{Generation of TIPRL-promoter reporter constructs}

The forward primer that was designed to clone the TIPRL-promoter region included an NheI site at its $5^{\prime}$ terminus and contained the following sequence: $5^{\prime}$-CCCGCTAGCTACCTGCAATTCCCAAC TCCC- $3^{\prime}$. The reverse primers included a HindIII site at its $5^{\prime}$ terminus and contained the following sequences: R1; 5'-CCCAAGCTTGGC TGAGGCAGGACCCGCAGA- $3^{\prime}$ and R2; 5'-CCCAAGCTTGAAGCA GAAATCCCGGTGGCT-3'. PCR was performed with LA Taq polymerase (Takara Shuzo), and PCR products were ligated into a T-vector and digested with $X \mathrm{cmI}$. The cloned promoter regions were then subcloned into a pGL2-basic vector (Promega, Madison, WI, USA) for the analysis of TIPRL-promoter activity. A 935-bp region of the TIPRL promoter was cloned into the pGL2-luciferase vector (TIPRL_R1). In addition, a 980-bp region of the TIPRL promoter with the ATG region was also cloned into the pGL2-luciferase vector (TIPRL_R2).

\section{Luciferase activity assay}

H1299 cells were transfected with plasmids that contained luciferase reporter genes (TIPRL_R1-pLuc and TIPRL_R2-pLuc) and the control Renilla-pLuc plasmid using Lipofectamine 2000 (Invitrogen). The Dual-Luciferase Reporter Assay System (Promega) was used to assay the luciferase activities after $24 \mathrm{~h}$ according to the manufacturer's instructions. At least three independent experiments were performed in duplicate, and the promoter activity was normalized to the Renilla activity.

\section{Data analysis}

All experiments were independently repeated at least three times, and the data are presented as the mean \pm standard error (s.e.). The non-parametric Mann-Whitney $U$-test was used to analyze the mean values, and $P$-values $<0.05$ were considered statistically significant.

\section{RESULTS}

cAMP signaling increased HDAC8 expression via a PKAindependent pathway

First, we confirmed the effect of cAMP signaling on HDAC8 expression by analyzing the HDAC8 protein after activation of cAMP signaling by expressing a constitutively active Gos (GosQL) or by treatment with Gos-coupled receptor agonists, such as ISO and prostaglandin E2, in human non-small cell lung cancer cells (H1299 and A549). The prostaglandin E2 treatment and the transient GosQL expression increased HDAC8 protein levels in H1299 cells (Figure 1a). Similarly, activation of cAMP signaling with ISO or by GosQL expression increased the HDAC8 protein levels in A549 cells (Figure 1b).
These results confirmed that cAMP signaling increased HDAC8 protein expression in human non-small cell lung cancer cells.

We then investigated the role of PKA, the best-known cAMP effector, in the CAMP-mediated increase in HDAC8 expression. PKA inhibition with a dominant negative PKA did not block the HDAC8-increasing effects of GosQL and ISO in H1299 cells (Figures 1c and d). The inhibition of PKA by treatment with a selective PKA inhibitor (H-89) did not block the HDAC8-increasing effect of ISO (Figure 1e). In addition, the activation of PKA by the expression of a PKA catalytic subunit did not significantly increase HDAC8 expression, though it increased phosphorylation of multiple PKA substrates (Figure 1f). These results indicate that cAMP signaling increases HDAC8 expression through a PKA-independent pathway.

\section{ISO increased HDAC8 expression via the Epac2-Rap1A pathway}

To identify the PKA-independent signaling pathway that mediated the HDAC8-increasing effect of cAMP, we assessed the role of another cAMP effector, Epac. The knockdown of Epacl using an Epac1-specific shRNA did not block the ISO-induced increase in HDAC8 expression, though it increased the unstimulated level of HDAC8 expression (Figure 2a). However, the knockdown of Epac2 by shRNA or with an Epac2-selective inhibitor (ESI-05) abolished the ISO-induced increase in HDAC8 expression (Figures 2b and c). Treatment with an Epac-selective agonist, 8-pCPT-2'$\mathrm{O}-\mathrm{Me}-\mathrm{cAMP}$, increased the HDAC8 protein levels to a degree similar to that observed in ISO-treated cells (Figure 2d), suggesting that cAMP signaling increases HDAC8 expression through an Epac2-dependent pathway. As a downstream target of Epac2 in ISO-induced HDAC8 expression, the involvement of Rap1A, a well-known effector of Epac, was examined. Treatments with ISO or 8-pCPT-2'-O-Me-cAMP increased GTP-bound Rap1, which indicated increased Rap1 activation (Figure 3a); Epac2 inhibition with an inhibitor (ESI-05) abolished the ISO-induced increase in GTP-bound Rap1 (Figure 3b). Rap1A activation via the expression of constitutively active Rap1A (caRap1A) also increased HDAC8 expression (Figure 3c). Moreover, Rap1A inhibition with a dominant negative Rap1A (dnRap1A) or through shRNA-mediated knockdown abolished the ISO-induced increase in HDAC8 expression (Figures $3 \mathrm{~d}$ and e). These results indicated that cAMP signaling increased HDAC8 protein expression via an Epac2-Rap1A-mediated pathway.

\section{ISO increased HDAC8 expression by inhibiting the PI3K/Akt pathway}

Because phosphatidylinositol 3-kinase (PI3K) and Akt are downstream signaling molecules of Rap1 in ISO-induced HDAC8 expression and because inhibition of PI3K and Akt inhibited JNK phosphorylation in our previous study, we tested their involvement here. ${ }^{13}$ Activation of cAMP signaling with ISO or 8-pCPT-2'-O-Me-cAMP inhibited the activating phosphorylations of Akt at Thr-308 and Ser-473 (Figure 4a). 

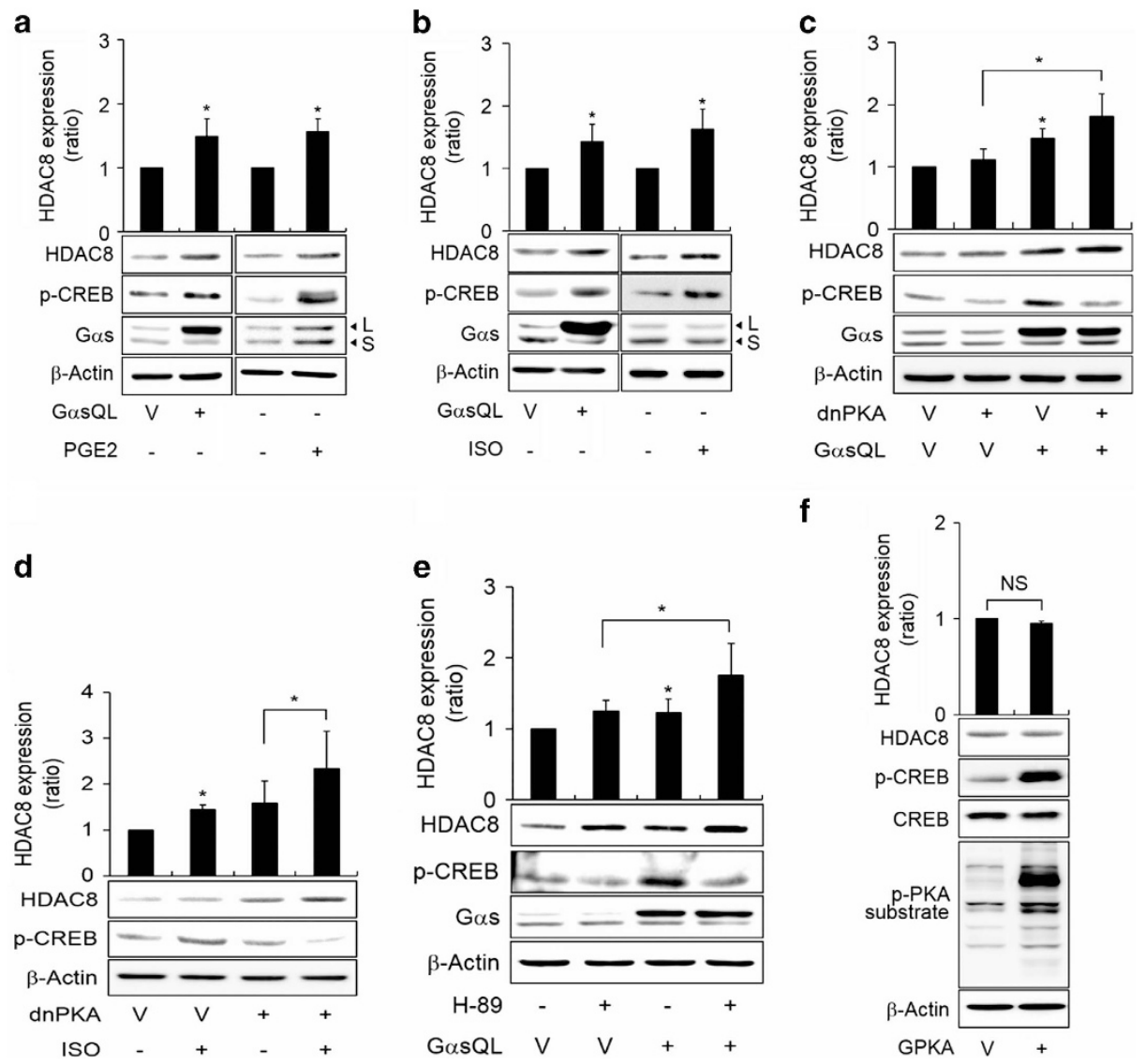

Figure 1 cAMP signaling increased HDAC8 expression via a PKA-independent pathway. (a) Effects of Gos or PGE2 on HDAC8 expression in H1299 lung cancer cells. (b) Effects of Gas or ISO on HDAC8 expression in A549 lung cancer cells. (c) Effects of PKA inhibition with dominant negative PKA (dnPKA) on GosQL-induced HDAC8 expression. (d) Effects of PKA inhibition with dnPKA on ISO-induced HDAC8 expression. (e) Effects of PKA inhibition with H-89 on GosQL-induced HDAC8 expression. (f) Effect of the catalytic subunit of PKA (GPKA) on HDAC8 expression. Non-small cell lung cancer cells (H1299 and A549) were transfected with EE-tagged GasQL (GosQL), dnPKA, GPKA or corresponding control vectors and incubated for $24 \mathrm{~h}$ (GosQL, GPKA) or $48 \mathrm{~h}$ (dnPKA) before treatments with $20 \mu \mathrm{m}$ ISO or $20 \mu \mathrm{m}$ PGE2 for $30 \mathrm{~min}$. The two arrowheads in the Gos blots indicate the long (L) and short $(\mathrm{S})$ forms of the Gos protein. The cells were pretreated with $20 \mu \mathrm{m} \mathrm{H}-89$ for $1 \mathrm{~h}$, followed by the ISO treatment and a subsequent western blot analysis. Asterisks (*) on the bar graphs indicate a statistically significant difference compared with the corresponding control $(P<0.05$, Mann-Whitney $U$-test).

Expression of a constitutively active Rap1A also decreased Akt phosphorylation at Thr-308 and Ser-473 (Figure 4b), and Rap1A knockdown restored the Akt phosphorylation from the ISO-induced decrease (Figure 4c). These results suggest that cAMP signaling inhibits the PI3K/Akt pathway in an Epac2-Rap1A-dependent manner.

To assess the roles of PI3K and Akt in the cAMP-induced increase in HDAC8 expression, the effects of PI3K and Akt on JNK activation and HDAC8 protein expression were examined. Inhibition of PI3K with PI3K inhibitors (LY294002 and wortmannin) inhibited the activating phosphorylations of MKK4 and JNK1 and increased the HDAC8 protein (Figure 5a). Expression of a dominant negative PI3K (dnPI3K) also increased HDAC8 expression (Figure 5b). Similarly, Akt inhibition with an Akt inhibitor (MK-2206) or by expression of a dominant negative Akt1 (dnAkt) inhibited the activating phosphorylations of MKK4 and JNK1 and increased the HDAC8 protein (Figures $5 \mathrm{c}$ and $\mathrm{d}$ ) without changing HDAC8 mRNA levels. Because Akt inhibition results in a reduction in inhibitory phosphorylation and subsequent activation of glyco-

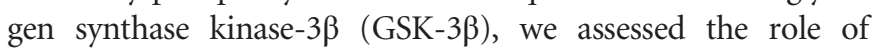
GSK-3 $\beta$ as a downstream mediator of Akt in the ISO-induced increase in HDAC8 expression. Inhibition of GSK-3 $\beta$ with GSK-3 $\beta$ inhibitors (CHIR99021 and lithium chloride) increased basal HDAC8 protein expression but did not block the ISO-induced increase in HDAC8 expression (Figures 5e and $f$ ), suggesting that GSK- $3 \beta$ did not mediate the cAMP effect on HDAC8 degradation. To confirm that Akt increased HDAC8 levels by inhibiting protein degradation, we analyzed the role of proteasomal or lysosomal degradation in the Akt inhibitor-induced increase in HDAC8 expression. The HDAC8 expression increases in the $\mathrm{H} 1299$ cells treated with lysosomal inhibitors $\left(\mathrm{HN}_{4} \mathrm{Cl}\right.$ and chloroquine) or a proteasomal inhibitor (MG-132) were similar to those treated with the Akt inhibitor (MK-2206). However, the MK-2206 treatment did not increase the HDAC8 protein levels above those observed in cells that were pretreated with the lysosomal or proteasomal inhibitors (Figure $5 \mathrm{~g}$ ). These findings indicate that the PI3K/Akt pathway 
a

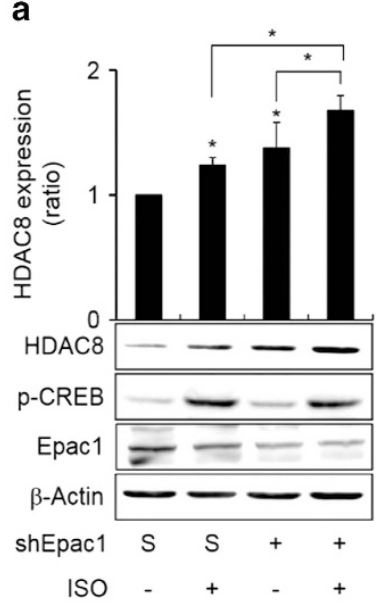

C

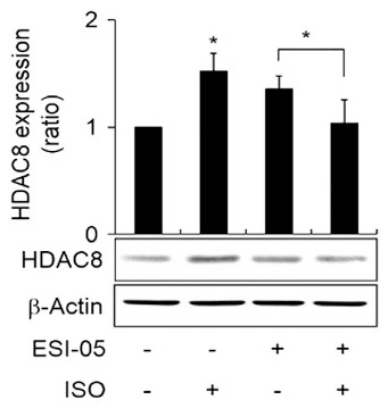

b

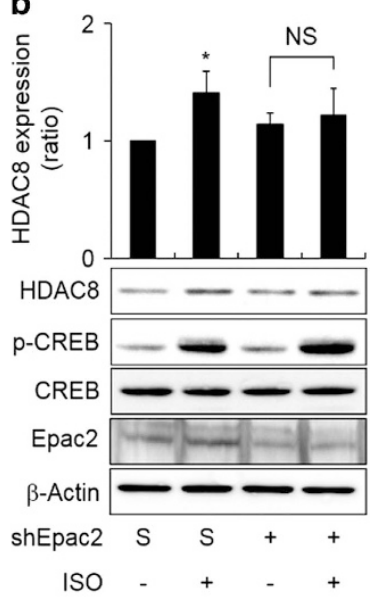

d

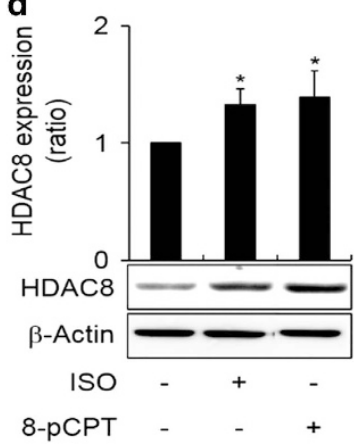

Figure 2 The Epac2 pathway mediated the ISO-induced increase in HDAC8 expression. (a) Effect of Epac1 knockdown on ISO-induced HDAC8 expression. (b) Effect of Epac2 knockdown on ISO-induced HDAC8 expression. (c) Effect of inhibiting Epac2 with a selective inhibitor (ESI-05) on ISO-induced HDAC8 expression. (d) Effect of an Epac-selective activator (8-pCPT-2'-O-Me-cAMP) on HDAC8 expression. H1299 cells were transfected with shRNA against Epac1 or Epac2 or with a scrambled shRNA (S) for $48 \mathrm{~h}$ before the $20 \mu \mathrm{m}$ ISO treatment for $30 \mathrm{~min}$ and the subsequent western blot analysis. The cells, which had been pretreated with or without $10 \mu \mathrm{m}$ ESI-05 for $30 \mathrm{~min}$, were treated with $20 \mu \mathrm{m}$ ISO or $20 \mu \mathrm{m}$ 8-pCPT-2'-0-Me-cAMP (8-pCPT) for $30 \mathrm{~min}$ before the western blot analysis. Asterisks (*) on the bar graphs indicate a statistically significant difference compared with the corresponding control or vector-transfected cells, and NS indicates no statistically significant difference compared with the corresponding control $(P<0.05$, Mann-Whitney $U$-test).

mediated the ISO-induced increase in HDAC8 expression by inhibiting activation of MKK4 and JNK1, which decreased HDAC8 protein degradation through the proteasome and by autophagy.

ISO augments cisplatin-induced apoptosis through HDAC8dependent repression of TIPRL expression in $\mathrm{H} 1299$ cells To study the physiological impact of the cAMP-induced HDAC8 expression increase, we examined the effect of the HDAC8 increase on anticancer drug- or ionizing radiationinduced apoptosis. Activation of cAMP signaling with ISO in cisplatin-treated H1299 cells increased the annexin V-stained

cell population and the cleavage of caspase- 3 and PARP, whereas HDAC8 knockdown with the HDAC8 shRNA abolished the apoptosis-augmenting effects of ISO (Figures 6a and b). Treatment with ISO also increased the cleavage of caspase-3 and PARP in $\gamma$-ray-irradiated H1299 cells, whereas HDAC8 knockdown abolished the apoptosis-augmenting effects of ISO (Figure 6c). These results indicated that cAMP signaling augmented cisplatin- or ionizing radiation-induced apoptosis in an HDAC8-dependent manner.

To explore the underlying mechanism by which HDAC8 augmented cisplatin-induced apoptosis, we analyzed the effect of HDAC8 on TIPRL expression because human TIPRL reportedly modulates TRAIL-induced apoptosis. ${ }^{16} \mathrm{We}$ found that its expression was reduced by cAMP signaling (our unpublished data). Treatment with cisplatin increased the TIPRL protein levels; exogenous HDAC8 expression decreased cisplatin-induced TIPRL protein expression and increased cisplatin-induced cleavage of caspase- 3 and caspase9 (Figure 6d). The siRNA-mediated knockdown of TIPRL increased the cleavage of caspase- 3 and PARP in cisplatintreated cells (Figure 6e), which demonstrated an anti-apoptotic role for TIPRL in H1299 cells. The ISO treatment also decreased TIPRL expression in cisplatin-treated H1299 cells; the HDAC8 knockdown with shRNA or siRNA abolished the ISO-induced decrease in TIPRL expression and the augmentation of caspase-9 and PARP cleavage (Figures $7 \mathrm{a}$ and $\mathrm{b}$ ).

To study the mechanism by which HDAC8 decreases TIPRL expression, we analyzed the effect of HDAC8 on TIPRL mRNA levels. The ISO treatment decreased TIPRL mRNA levels in cisplatin-treated H1299 cells, whereas the HDAC8 knockdown blocked the ISO-induced mRNA decrease, suggesting that ISO decreased the TIPRL mRNA levels via a HDAC8-dependent mechanism (Figure 7c). To examine whether the decrease in the TIPRL mRNA levels resulted from a decrease in TIPRL gene transcription, we analyzed the activity of luciferase reporters that contained TIPRL-promoter sequences. The cisplatin treatment increased the luciferase activities of the two reporters that contained the TIPRL-promoter regions (TIPRL_R1-pLuc and TIPRL_R2-pLuc), and the ISO treatment decreased the cisplatin-induced luciferase activity. However, the HDAC8 knockdown blocked the ISO-induced decrease in cisplatin-induced luciferase activity (Figure $7 \mathrm{c}$ and data now shown). To examine the effects of HDAC8-specific inhibitors on TIPRL expression, we treated the cells with PCI-34051 or NCC-149. The inhibitor treatments did not abolish the ISO effects on TIPRL expression and caspase-9 cleavage (Figures 7d and e). These results indicate that ISO augments cisplatininduced apoptosis by repressing TIPRL transcription in an HDAC8-dependent manner in H1299 cells.

\section{DISCUSSION}

This study was performed to investigate the signaling pathway that mediates CAMP-induced inhibition of HDAC8 degradation and the effects and underlying mechanisms of the resulting increase in HDAC8 expression on cisplatin-induced apoptosis in lung cancer cells. We found that cAMP signaling inhibits 
a

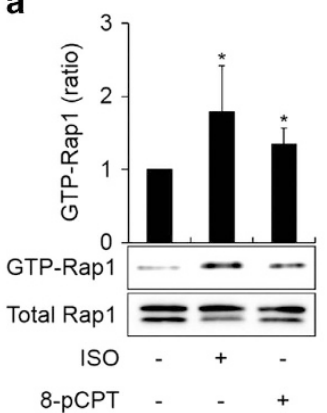

b

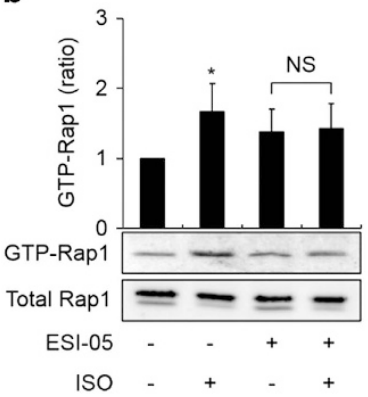

C

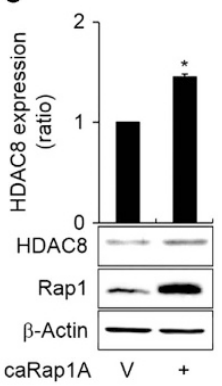

d

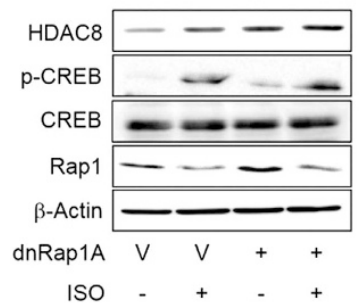

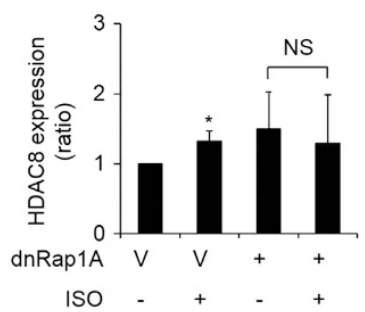

e
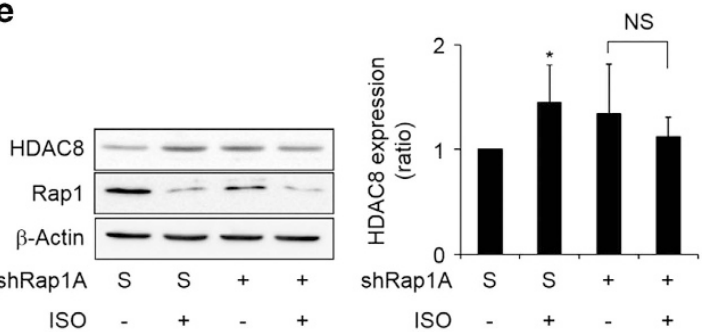

Figure 3 The Rap1A pathway mediated the ISO-induced increase in HDAC8 expression. (a) Effects of ISO or 8-pCPT-cAMP on Rap1 activation. (b) Effects of inhibiting Epac2 with a selective inhibitor (ESI-05) on ISO-induced GTP-bound Rap1. H1299 cells were pretreated with $10 \mu \mathrm{m}$ ESI-05 or vehicle for $30 \mathrm{~min}$ and then treated with $20 \mu \mathrm{m}$ ISO or $20 \mu \mathrm{m} 8$-pCPT-2'-0-Me-cAMP (8-pCPT) for 30 min before the pull-down assay. (c) Effect of Rap1A activation on HDAC8 expression. (d) Effect of dominant negative Rap1A on ISO-induced HDAC8 expression. (e) Effect of Rap1A knockdown on ISO-induced HDAC8 expression. Cells were transfected with constitutively active Rap1A-V12 (caRap1A), dominant negative Rap1A-N17 (dnRap1A), shRNA against Rap1A, or with the corresponding control vector (V) and incubated for $48 \mathrm{~h}$ before treatment with $20 \mu \mathrm{M}$ ISO for 30 min and a subsequent western blot analysis. Asterisks $\left({ }^{*}\right)$ on the bar graphs indicate a statistically significant difference compared with the corresponding control or vector-transfected cells, and NS indicates no statistically significant difference compared with the corresponding control $(P<0.05$, Mann-Whitney U-test).

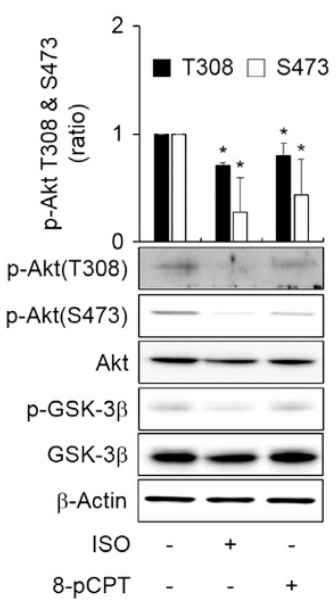

b

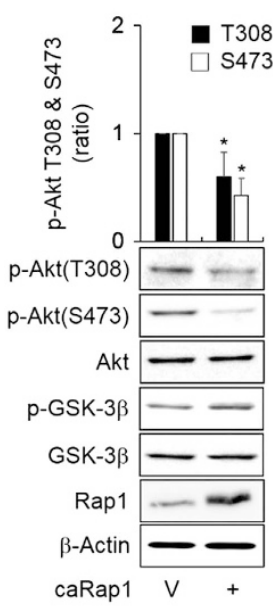

c

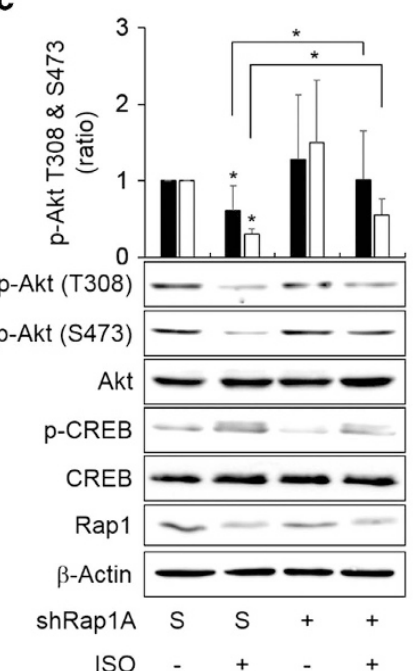

Figure 4 The Rap1A pathway mediated the ISO-induced inhibition of Akt. (a) Effects of ISO or 8-pCPT-2'-O-Me-cAMP on Akt activation. (b) Effects of Rap1 activation on Akt activation. (c) Effects of Rap1A knockdown on Akt activation. H1299 cells were transfected with constitutively active Rap1A (caRap1A), shRNA against Rap1A (shRap1A), vector (V) or scrambled shRNA (S) and incubated for 24 h for caRap1A or $48 \mathrm{~h}$ for shRap1A before the ISO treatment. H1299 cells were treated with $20 \mu \mathrm{m}$ ISO or $20 \mu \mathrm{m} 8$-pCPT-cAMP for 30 min before the western blot analysis. Asterisks $\left({ }^{*}\right)$ on the bar graphs indicate a statistically significant difference compared with the corresponding control $(P<0.05$, Mann-Whitney $U$ test). 
a

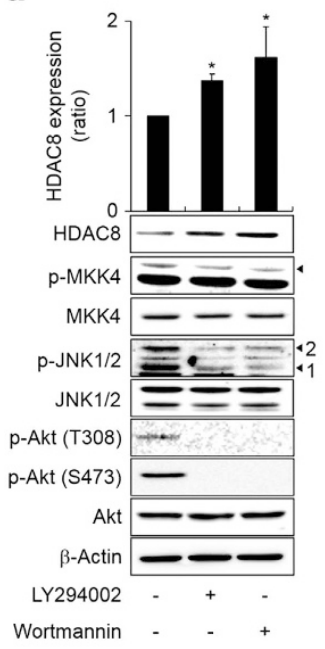

e

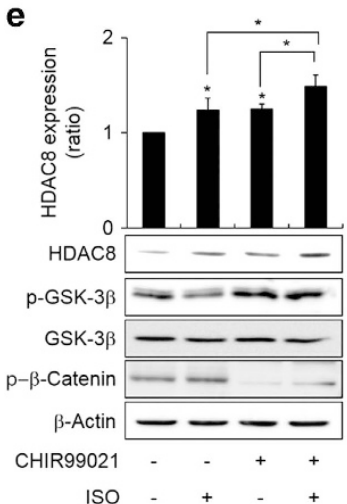

b

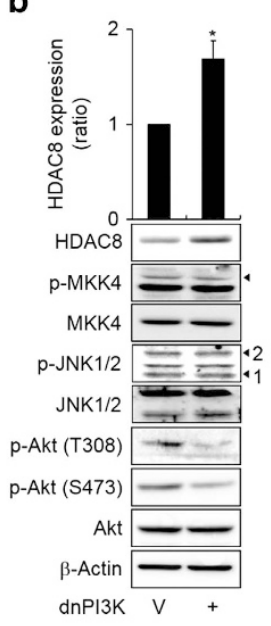

c

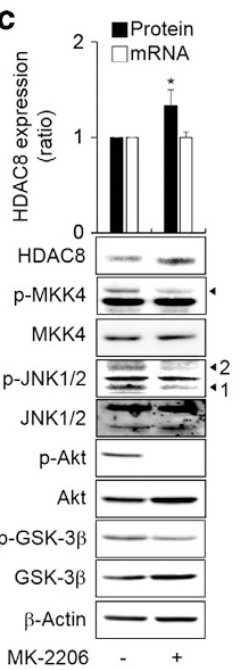

d

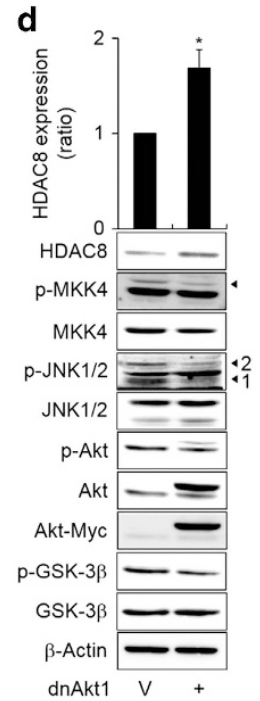

f

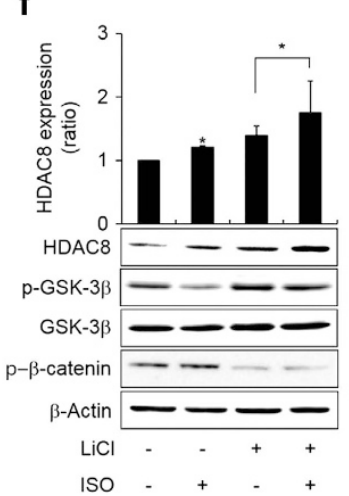

g

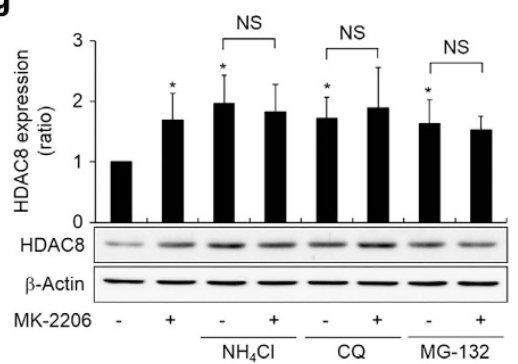

Figure 5 The PI3K/Akt pathway mediated the ISO-induced increase in HDAC8 expression. (a) Effects of PI3K inhibitors on HDAC8 expression. (b) Effects of dominant negative PI3K on HDAC8 expression. (c) Effects of an Akt inhibitor on HDAC8 expression. (d) Effects of dominant negative Akt on HDAC8 expression. (e, f) Effects of a GSK-3 $\beta$ inhibitor on ISO-induced HDAC8 expression. (g) Effects of lysosomal or proteasomal inhibitors on MK-2206-induced HDAC8 expression. H1299 cells were pretreated with $20 \mu \mathrm{m}$ chloroquine, 10 mm $\mathrm{NH} 4 \mathrm{Cl}$ or $10 \mu \mathrm{m} \mathrm{MG-132}$ for $4 \mathrm{~h}$ before the $10 \mu \mathrm{m}$ MK-2206 treatment for $30 \mathrm{~min}$. Cells were treated with $10 \mu \mathrm{m}$ LY294002, $100 \mathrm{nM}$ wortmannin, $10 \mu \mathrm{M}$ MK-2206, $10 \mu \mathrm{m} \mathrm{CHIR99021} \mathrm{or} 10 \mathrm{~mm} \mathrm{LiCl}$ for $30 \mathrm{~min}$ and subsequently collected and analyzed by western blot or qPCR. Cells were transfected with dominant negative PI3K (dnPI3K), dominant negative Akt1 (dnAkt1), or the corresponding control vector (V) and incubated for $24 \mathrm{~h}$ before analysis. Asterisks $\left(^{*}\right)$ on the bar graphs indicate a statistically significant difference compared with the corresponding control or vector-transfected cells, and NS indicates no statistically significant difference compared with the corresponding control $(P<0.05$, Mann-Whitney $U$ test).

HDAC8 protein degradation through Epac2-Rap1A-mediated inhibition of the PI3K/Akt pathway and that cAMP signaling augments cisplatin-induced apoptosis via HDAC8-dependent repression of TIPRL expression in H1299 non-small cell lung cancer cells (Figure 7f).

An increase in cAMP concentration activates three major effector molecules: PKA, Epac and cyclic nucleotide-gated ion channels. These effectors regulate diverse physiologic processes, including metabolism, gene expression, proliferation, differentiation and cell death. ${ }^{2}$ Thus, we studied which of the cAMP effectors mediates the increase in HDAC8 expression and found that Epac2-Rap1A, but not PKA, mediates HDAC8 expression. This finding was supported by the observations that the Epac-selective cAMP analog increased HDAC8 expression and that the ISO effect was abolished when Epac2 was inhibited by a specific inhibitor or by knockdown. However, the Epac1 knockdown did not abolish the ISO effect, which indicated that the HDAC8 expression increase was Epac2-specific. Epac is a cAMP-regulated guanine nucleotide exchange factor for the direct activation of the Rap1 and Rap2 Ras family members. ${ }^{17,18}$ Epac proteins regulate various biological functions, including cell proliferation, differentiation, survival and apoptosis. Thus, alterations to Epac signaling have been implicated in the pathophysiologies of cancers and numerous diseases, including those of the heart, brain and lung. ${ }^{19}$ Epac activation reportedly induces the nuclear export of HDAC4, which suggests its role in epigenetic regulation. ${ }^{20}$ Our study presents a new mechanism by which Epac epigenetically regulates gene expression by increasing the expression of the HDAC8 protein. 
a

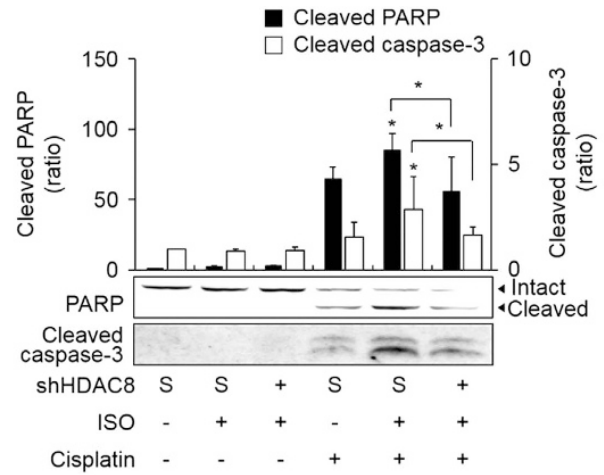

b

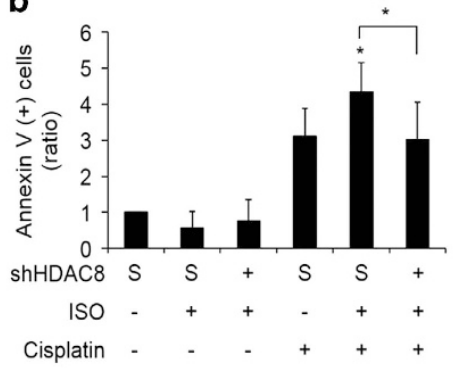

C

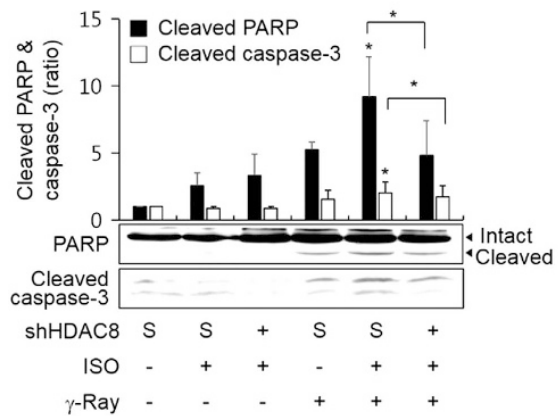

d

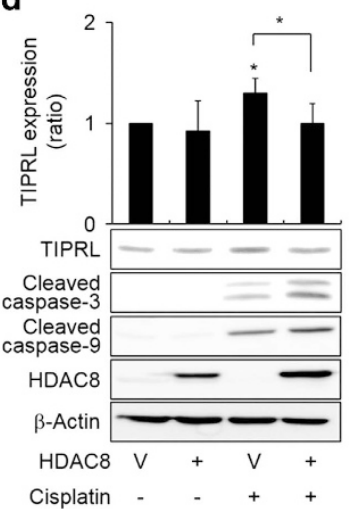

e Cleaved PARP

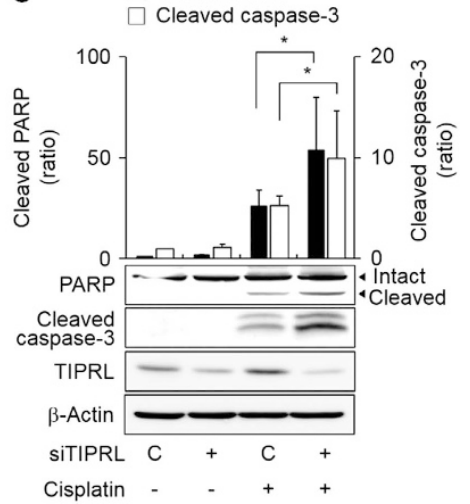

Figure 6 ISO augmented cisplatin-induced apoptosis by increasing HDAC8 expression. (a) Effects of ISO and HDAC8 on cisplatin-induced cleavage of caspase-3 and PARP. (b) Effects of ISO and HDAC8 on cisplatin-induced annexin V-staining. (c) Effects of ISO and HDAC8 on $\gamma$-ray-induced cleavage of caspase-3 and PARP. (d) Effects of HDAC8 on cisplatin-induced TIPRL expression. (e) Effects of TIPRL knockdown on cisplatin-induced cleavage of caspase-3 and PARP. H1299 cells were transfected with wild-type HDAC8, HDAC8 shRNA, scrambled shRNA (S), TIPRL siRNA, control siRNA (C) or the corresponding control vectors (V) and incubated for 24 h. The cells were pretreated with ISO for 30 min (if necessary) and treated with $50 \mu \mathrm{m}$ cisplatin. Asterisks $\left(^{*}\right)$ on the bar graphs indicate a statistically significant difference compared with the corresponding control $(P<0.05$, Mann-Whitney $U$-test).

We also analyzed whether Rap1, which is an immediate downstream Epac target, mediated the ISO-induced increase in HDAC8 expression and found that Rap1A mediated the induction. This mediation was evidenced by the findings that Rap1 was activated by ISO, that activation of Rap1A increased HDAC8 expression and that inhibition of Rap1A abolished the ISO-induced HDAC8 expression increase. The Rap proteins belong to the Ras family of small G proteins, and several Rap isoforms are expressed in mammals. Rap proteins are activated by Rap guanine nucleotide exchange factors, including Epacs, and Rap1 is specifically activated by an Epac2-mediated cAMP signaling pathway. ${ }^{21}$ Rap proteins are implicated in various biological processes, including cell proliferation, differentiation and apoptosis, ${ }^{22}$ but the role of Rap in the deacetylation modification is unclear. Therefore, our finding that Rap1 mediates the HDAC8 expression increase suggests that the Rap1 protein might have a role in epigenetic regulation by modulating histone acetylation.

During our attempt to discover the signaling pathway acting downstream of Epac2-Rap1A, we found that PI3K/Akt pathways is inhibited through Epac2-Rap1A and mediates the cAMP signaling-induced decrease in HDAC8 degradation by inhibiting JNK pathways. This finding was supported by the observations that activation of cAMP signaling inhibited Akt activity via Rap1A and that inhibition of PI3K/Akt with specific pharmacological inhibitors or by expressing dominant negative enzymes inhibited the MKK4/JNK pathway and HDAC8 protein degradation. We recently reported that cAMP signaling increased HDAC8 expression by inhibiting JNK-dependent degradation of the HDAC8 protein via autophagy and the proteasome system in lung cancer cells. ${ }^{13}$ Thus, this study shows that PI3K/Akt acts upstream of the MKK4/JNK pathway and downstream of Epac/Rap1 to mediate the cAMP-induced inhibition of HDAC8 degradation. Our finding is consistent with the observations that cAMP signaling inhibits Akt activity by activating Epac and Rap1 in thyroid and kidney cells ${ }^{23-25}$ and that the Rap1 protein acts as a negative regulator of the PI3K/Akt pro-survival signaling pathway in B cells through a mechanism that involves the binding of Rap-GTP to PI3K. ${ }^{26}$ However, Epac reportedly increases Akt activity in neuronal cells and mesenchymal stem cells. ${ }^{27}$ The mechanism for the differential effects of cAMP/Epac2/Rap1 on Akt activation is unclear but might shed light on a potential mechanism for the cell type-specific effects of cAMP signaling.

Akt regulates the two major intracellular proteolytic signaling pathways-ubiquitination and autophagy ${ }^{28}$-and is 
a

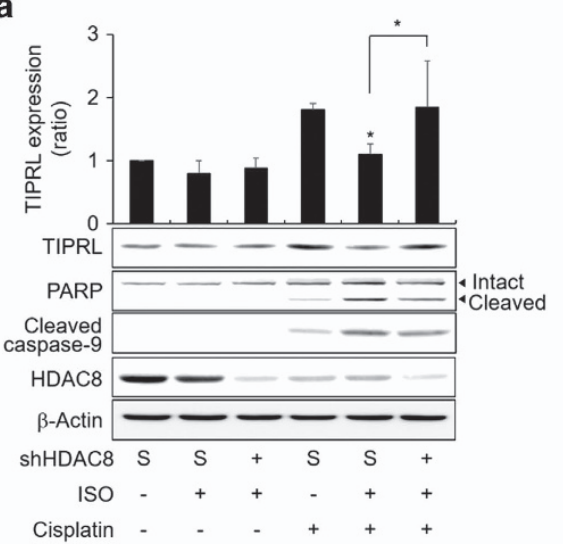

c

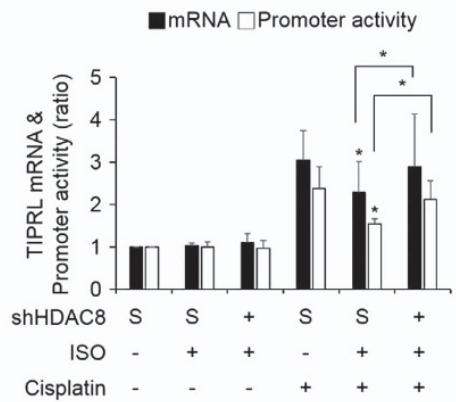

e

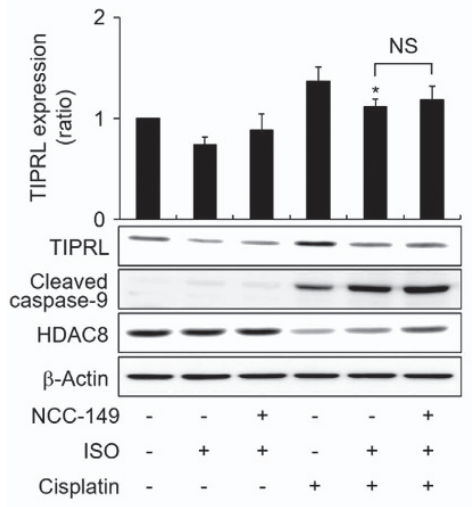

b

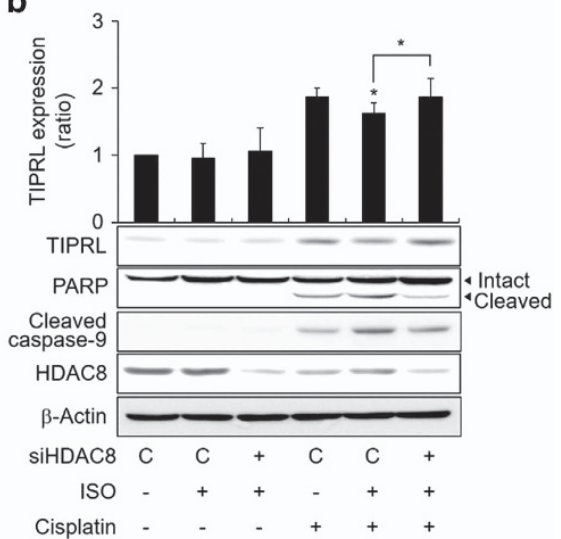

d

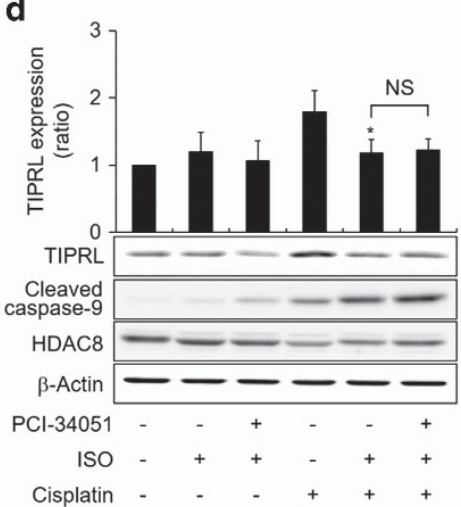

f

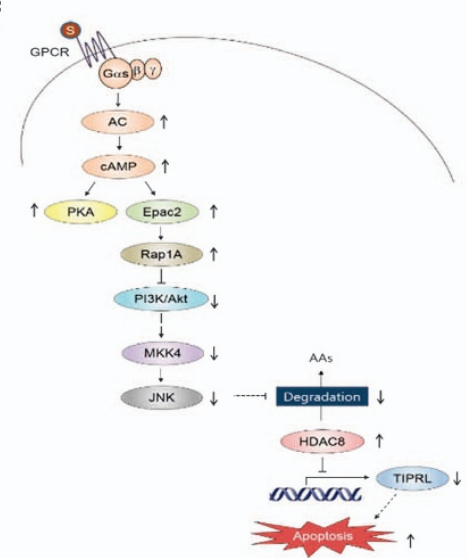

Figure 7 ISO augmented cisplatin-induced apoptosis by repressing TIPRL expression in a HDAC8-dependent pathway. (a, b) Effects of HDAC8 knockdown on ISO-induced augmentation of apoptosis and TIPRL expression. H1299 cells were transfected with HDAC8 shRNA, scrambled shRNA (S), HDAC8 siRNA, or a control siRNA (C) and incubated for $24 \mathrm{~h}$. The transfected cells were pretreated with ISO for $30 \mathrm{~min}$ and subsequently treated with $50 \mu \mathrm{m}$ cisplatin for $48 \mathrm{~h}$ before the western blot analysis. (c) Effects of ISO and HDAC8 on the TIPRL mRNA level and TIPRL-promoter activity in cisplatin-treated cells. H1299 cells were transfected with TIPRL-luciferase constructs (Renilla-pLuc and TIPRL_R1-pLuc that contained a 935- bp region of the TIPRL promoter) or the corresponding control vectors (V) and treated with $50 \mu \mathrm{m}$ cisplatin for $24 \mathrm{~h}$ before the qPCR or luciferase assay. (d, e) Effects of HDAC8 activity on ISO-induced augmentation of apoptosis and TIPRL expression. H1299 cells were sequentially treated with $10 \mu \mathrm{m} \mathrm{PCl-34051} \mathrm{or} 2 \mu \mathrm{m}$ NCC-149 for $24 \mathrm{~h}$, ISO for 30 min and $50 \mu \mathrm{m}$ cisplatin for $48 \mathrm{~h}$ before the western blot analysis. (f) A proposed mechanism by which cAMP signaling inhibits Akt to decrease HDAC8 degradation, which augments apoptosis in lung cancer cells. S represents a signal, and AAs represent amino acids. The solid lines indicate proven signaling pathways, and the dotted lines indicate suggested signaling pathways. Asterisks $\left(^{*}\right)$ on the bar graphs indicate a statistically significant difference compared with the corresponding control, and NS indicates no statistically significant difference compared with the corresponding control $(P<0.05$, Mann-Whitney $U$-test). 
involved in the degradation of numerous proteins. ${ }^{29} \mathrm{PI} 3 \mathrm{~K} / \mathrm{Akt}$ has been reported to mediate laminar flow-induced activation of histone deacetylases, including HDAC8, in endothelial cells, ${ }^{30}$ but the role of Akt on HDAC8 expression remained unclear. Our studies present a novel role for PI3K/Akt in the regulation of HDAC8 degradation through JNK pathway activation, which reportedly phosphorylates the Itch E3 ubiquitin ligase (to stimulate the ubiquitin/proteasome system) and $\mathrm{Bcl}-2$ (to stimulate autophagy for HDAC8 protein degradation) in H1299 lung cancer cells. ${ }^{13}$ Our finding suggests the activation of MKK4/JNK, which is involved in controlling protein degradation, as a novel mechanism for Akt-mediated regulation of protein degradation. Activation of the JNK pathway by Akt, whereby inhibition of Akt activation blocks anti-5-methylchrysene-1,2-diol-3,4-epoxide (5-MCDE)induced activation of JNKs in mouse epidermal cells, has been previously described. ${ }^{31}$ However, the upstream signaling molecules for MKK4-mediated JNK activation by Akt are unknown. cAMP signaling regulates both the proteasomal and lysosomal degradation of various proteins. For example, cAMP-Epac1 signaling regulates the $\mathrm{ECS}^{\text {socs-3 }} \mathrm{E} 3$ ubiquitin ligase complex $\mathrm{t}$ o regulate cytokine signaling, ${ }^{32}$ and cAMP signaling inhibits autophagy through the cAMP-Epac-phospholipase C pathway. ${ }^{33}$ This study presents inhibition of the JNK pathway via Epac2-Rap1A-Akt as a novel pathway for cAMP signaling to regulate protein degradation in non-small cell lung cancer cells.

In contrast to Epac, our results indicate that PKA does not mediate the ISO-induced increase in HDAC8 expression. Blocking PKA did not abolish the HDAC8 expression increase that was induced by activation of cAMP signaling, and activation of PKA did not increase HDAC8 expression, though it did increase the phosphorylation of multiple PKA target proteins, including CREB. However, PKA has been reported to phosphorylate HDAC8 with a consequent reduction in its activity in HeLa cervical cancer cells. ${ }^{34}$ Therefore, it is possible that cAMP signaling modulates HDAC8 activity either through inhibitory phosphorylation by PKA or by HDAC8 protein upregulation through Epac. However, the mechanism for the regulation of the two dichotomous cAMP signaling pathways is unclear and needs to be elucidated.

Inhibition of Akt with ISO decreases the inhibitory phosphorylation of GSK-3 $\beta$, which activates the enzyme. Therefore, GSK-3 $\beta$ was assessed to determine if it mediated the ISO-induced increase in HDAC8 expression. Inhibition of GSK-3 $\beta$ activity with inhibitors did not block the ISO-induced increase in HDAC8 expression, which indicated that ISO increased HDAC8 expression through a GSK-3 $\beta$-independent pathway. However, the inhibition of GSK-3 $\beta$ activity alone increased HDAC8 expression, suggesting that GSK-3 $\beta$ might be involved in repressing basal HDAC8 expression.

To assess the physiological significance of cAMP-induced HDAC8 expression, we analyzed the effect of HDAC8 on apoptosis and investigated the underlying mechanism. We found that cAMP signaling augmented cisplatin-induced apoptosis by increasing HDAC8 expression, and the increase in HDAC8 expression augmented apoptosis by repressing TIPRL expression in H1299 lung cancer cells. This finding was corroborated by the observation that cAMP signaling activation augmented cisplatin- and $\gamma$-ray-induced apoptosis with a concomitant increase in HDAC8 expression. It was also consistent with the observation that exogenous HDAC8 expression resulted in an augmentation of cisplatin-induced apoptosis, whereas the HDAC8 knockdown abolished the apoptotic augmentation. Furthermore, HDAC8 reduced cisplatin-induced TIPRL expression, and the knockdown of TIPRL expression augmented cisplatin-induced apoptosis. Finally, cAMP signaling decreased TIPRL gene transcription in a HDAC8-dependent manner.

We previously reported that the cAMP signaling system regulated lung cancer cell apoptosis through various mechanisms (that is, by modulating the expression of $\mathrm{Bcl}-2$ family proteins $^{35,36}$ and by regulating ataxia-telangiectasia-mutated (ATM) activation in lung cancer cell ${ }^{37}$ ). Here, we introduce the repression of TIPRL expression by a HDAC8 expression increase as a novel mechanism by which cAMP signaling modulates apoptosis. However, repression of TIPRL expression by HDAC8 appeared to be independent of HDAC8 deacetylase activity because pharmacological inhibition of the HDAC8 activity (PCI-34051 and NCC-149), in contrast to HDAC8 knockdown with shRNA or siRNA, did not block the ISO-induced TIPRL repression or apoptotic augmentation. A similar effect for PCI-34051 on apoptosis was previously reported, whereby the PCI-34051-mediated inhibition of HDAC8 induced caspase-dependent apoptosis in cell lines that were derived from T-cell lymphomas or leukemias but not in other hematopoietic or solid tumor lines, including A549 lung cancer cells. ${ }^{14}$ The mechanisms that drive the different outcomes for HDAC8 expression versus activity are unclear, but it is likely that the HDAC8 protein might perform a function that is independent of its histone deacetylase activity during its regulation of TIPRL expression. Further studies are needed to elucidate the mechanism of this difference.

TIPRL, the mammalian orthologue of the yeast TIP41 protein, is reportedly present at higher levels in cancer tissues versus normal tissues, and the TIPRL knockdown augments TRAIL stimulation-induced apoptosis in hepatocellular carcinoma cell lines. ${ }^{16,38}$ These reports confirm our finding that HDAC8-dependent repression of TIPRL expression by cAMP signaling contributes to the augmentation of cisplatin-induced apoptosis. TIPRL also influences the phosphorylation state of a specific protein substrate of ATM- and Rad3-related (ATR) kinases, which implies a role for TIPRL in DNA repair ${ }^{39}$ that may contribute to the modulation of apoptosis. We found that HDAC8 inhibits TIPRL gene transcription, but the molecular mechanism for this transcriptional regulation needs to be studied further.

This study suggests that cAMP signaling can epigenetically regulate gene expression by controlling HDAC8 expression and by regulating the activities of transcription factors, including CREB. HDAC8 also deacetylates non-histone proteins that are involved in lung cancer cell proliferation ${ }^{40}$ and breast cancer 
cell invasion. ${ }^{41}$ Therefore, in addition to apoptosis, the biological effects of the cAMP-induced increase in HDAC8 expression need to be studied further. ISO is an isopropyl analog of epinephrine, a major stress hormone, that activates $\beta$-adrenergic receptors to activate cAMP signaling. $\beta$-adrenergic signaling regulates multiple cellular processes that contribute to the initiation and progression of cancer. ${ }^{42}$ Therefore, the targeting of cAMP signaling and HDACs has been investigated with the aim of developing novel approaches to enhance the therapeutic effects of various cancer treatments. ${ }^{4,43}$ Thus, this study might contribute to the development of new strategies that improve the efficacies of chemotherapy and radiotherapy against cancers, including lung cancers, by modulating the activities of cAMP signaling and HDACs.

In conclusion, this study shows that the Epac-Rap1-Akt pathway mediates cAMP signaling-induced inhibition of JNK-dependent HDAC8 degradation and that the resulting increase in HDAC8 augments cisplatin-induced apoptosis by repressing TIPRL expression in H1299 lung cancer cells.

\section{CONFLICT OF INTEREST}

The authors declare no conflict of interest.

\section{ACKNOWLEDGEMENTS}

This work was supported by a grant from the National R\&D Program for Cancer Control, Ministry of Health and Welfare, Republic of Korea (0720540), by the Basic Science Research Program through the National Research Foundation funded by the Ministry of Education, Science and Technology (2012R1A1A2044374) and by the Education and Research Encouragement Fund of Seoul National University Hospital.

1 Fimia GM, Sassone-Corsi P. Cyclic AMP signalling. J Cell Sci 2001; 114: 1971-1972.

2 Gold MG, Gonen T, Scott JD. Local cAMP signaling in disease at a glance. J Cell Sci 2013; 126: 4537-4543.

3 Savai R, Pullamsetti SS, Banat GA, Weissmann N, Ghofrani HA, Grimminger $\mathrm{F}$ et al. Targeting cancer with phosphodiesterase inhibitors. Expert Opin Investig Drugs 2010; 19: 117-131.

4 Murray F, Insel PA. Targeting cAMP in chronic lymphocytic leukemia: a pathway-dependent approach for the treatment of leukemia and lymphoma. Expert Opin Ther Targets 2013; 17: 937-949.

5 de Ruijter AJ, van Gennip AH, Caron HN, Kemp S, van Kuilenburg AB. Histone deacetylases (HDACs): characterization of the classical HDAC family. Biochem J 2003; 370: 737-749.

6 Barneda-Zahonero B, Parra M. Histone deacetylases and cancer. Mol Oncol 2012; 6: 579-589.

7 Buggy JJ, Sideris ML, Mak P, Lorimer DD, McIntosh B, Clark JM. Cloning and characterization of a novel human histone deacetylase, HDAC8. Biochem J 2000; 350: 199-205.

8 Karolczak-Bayatti M, Sweeney M, Cheng J, Edey L, Robson SC, Ulrich SM et al. Acetylation of heat shock protein 20 (Hsp20) regulates human myometrial activity. J Biol Chem 2011; 286: 34346-34355.

9 Wilson BJ, Tremblay AM, Deblois G, Sylvain-Drolet G, Giguere V. An acetylation switch modulates the transcriptional activity of estrogen-related receptor alpha. Mol Endocrinol 2010; 24: 1349-1358.

10 Chakrabarti A, Oehme I, Witt O, Oliveira G, Sippl W, Romier C et al. HDAC8: a multifaceted target for therapeutic interventions. Trends Pharmacol Sci 2015; 36: 481-492.

11 Laurent AC, Bisserier M, Lucas A, Tortosa F, Roumieux M, De Regibus A et al. Exchange protein directly activated by CAMP 1 promotes autophagy during cardiomyocyte hypertrophy. Cardiovasc Res 2015; 105: 55-64.
12 Lokireddy S, Kukushkin NV, Goldberg AL. cAMP-induced phosphorylation of 26S proteasomes on Rpn6/PSMD11 enhances their activity and the degradation of misfolded proteins. Proc Natl Acad Sci USA 2015; 112 E7176-E7185.

13 Park JY, Juhnn YS. cAMP signaling increases histone deacetylase 8 expression by inhibiting JNK-dependent degradation via autophagy and the proteasome system in $\mathrm{H} 1299$ lung cancer cells. Biochem Biophys Res Commun 2016; 470: 336-342.

14 Balasubramanian S, Ramos J, Luo W, Sirisawad M, Verner E, Buggy JJ. A novel histone deacetylase 8 (HDAC8)-specific inhibitor PCI-34051 induces apoptosis in T-cell lymphomas. Leukemia 2008; 22: 1026-1034.

15 Seo M, Cho CH, Lee YI, Shin EY, Park D, Bae CD et al. Cdc42-dependent mediation of UV-induced p38 activation by $\mathrm{G}$ protein betagamma subunits. J Biol Chem 2004; 279: 17366-17375.

16 Song IS, Jun SY, Na HJ, Kim HT, Jung SY, Ha GH et al. Inhibition of MKK7-JNK by the TOR signaling pathway regulator-like protein contributes to resistance of HCC cells to TRAIL-induced apoptosis. Gastroenterology 2012; 143: 1341-1351.

17 de Rooij J, Zwartkruis FJ, Verheijen MH, Cool RH, Nijman SM, Wittinghofer A et al. Epac is a Rap1 guanine-nucleotide-exchange factor directly activated by cyclic AMP. Nature 1998; 396: 474-477.

18 Kawasaki H, Springett GM, Mochizuki N, Toki S, Nakaya M, Matsuda M et al. A family of CAMP-binding proteins that directly activate Rap1. Science 1998; 282: 2275-2279.

19 Schmidt M, Dekker FJ, Maarsingh H. Exchange protein directly activated by cAMP (epac): a multidomain cAMP mediator in the regulation of diverse biological functions. Pharmacol Rev 2013; 65: 670-709.

20 Liu Y, Schneider MF. Opposing HDAC4 nuclear fluxes due to phosphorylation by beta-adrenergic activated protein kinase A or by activity or Epac activated CaMKII in skeletal muscle fibres. J Physiol 2013; 591: 3605-3623.

21 Li Y, Asuri S, Rebhun JF, Castro AF, Paranavitana NC, Quilliam LA. The RAP1 guanine nucleotide exchange factor Epac2 couples cyclic AMP and Ras signals at the plasma membrane. J Biol Chem 2006; 281: 2506-2514.

22 Gloerich M, Bos JL. Regulating Rap small G-proteins in time and space. Trends Cell Biol 2011; 21: 615-623.

23 Hong K, Lou L, Gupta S, Ribeiro-Neto F, Altschuler DL. A novel EpacRap-PP2A signaling module controls CAMP-dependent Akt regulation. J Biol Chem 2008; 283: 23129-23138.

24 Kim S, Jee K, Kim D, Koh H, Chung J. Cyclic AMP inhibits Akt activity by blocking the membrane localization of PDK1. J Biol Chem 2001; 276: 12864-12870.

25 Lou L, Urbani J, Ribeiro-Neto F, Altschuler DL. cAMP inhibition of Akt is mediated by activated and phosphorylated Rap1b. J Biol Chem 2002; 277: 32799-32806.

26 Christian SL, Lee RL, McLeod SJ, Burgess AE, Li AH, Dang-Lawson M et al. Activation of the Rap GTPases in B lymphocytes modulates B cell antigen receptor-induced activation of Akt but has no effect on MAPK activation. J Biol Chem 2003; 278: 41756-41767.

27 Jang MW, Yun SP, Park JH, Ryu JM, Lee JH, Han HJ. Cooperation of Epac1/Rap1/Akt and PKA in prostaglandin E(2) -induced proliferation of human umbilical cord blood derived mesenchymal stem cells: involvement of c-Myc and VEGF expression. J Cell Physiol 2012; 227: 3756-3767.

28 Noguchi M, Hirata N, Suizu F. The links between AKT and two intracellular proteolytic cascades: ubiquitination and autophagy. Biochim Biophys Acta 2014; 1846: 342-352.

29 Heras-Sandoval D, Perez-Rojas JM, Hernandez-Damian J, Pedraza-Chaverri J. The role of PI3K/AKT/mTOR pathway in the modulation of autophagy and the clearance of protein aggregates in neurodegeneration. Cell Signal 2014; 26: 2694-2701.

30 Lee DY, Lee Cl, Lin TE, Lim SH, Zhou J, Tseng YC et al. Role of histone deacetylases in transcription factor regulation and cell cycle modulation in endothelial cells in response to disturbed flow. Proc Natl Acad Sci USA 2012; 109: 1967-1972.

$31 \mathrm{Li} \mathrm{DQ}$, Chen Z, Song XJ, Luo L, Pflugfelder SC. Stimulation of matrix metalloproteinases by hyperosmolarity via a JNK pathway in human corneal epithelial cells. Invest Ophthalmol Vis Sci 2004; 45: 4302-4311.

32 Williams JJ, Palmer TM. Unbiased identification of substrates for the Epac1-inducible E3 ubiquitin ligase component SOCS-3. Biochem Soc Trans 2012; 40: 215-218.

33 Fleming A, Noda T, Yoshimori T, Rubinsztein DC. Chemical modulators of autophagy as biological probes and potential therapeutics. Nat Chem Biol 2011; 7: 9-17. 
34 Lee H, Rezai-Zadeh N, Seto E. Negative regulation of histone deacetylase 8 activity by cyclic AMP-dependent protein kinase A. Mol Cell Biol 2003; 24: 765-773.

35 Choi YJ, Oh JM, Kim SY, Seo M, Juhnn YS. Stimulatory heterotrimeric GTPbinding protein augments cisplatin-induced apoptosis by upregulating Bak expression in human lung cancer cells. Cancer Sci 2009; 100: 1069-1074.

36 Choi YJ, Kim SY, Oh JM, Juhnn YS. Stimulatory heterotrimeric G protein augments gamma ray-induced apoptosis by up-regulation of Bak expression via CREB and AP-1 in H1299 human lung cancer cells. Exp Mol Med 2009; 41: 592-600.

37 Cho EA, Kim EJ, Kwak SJ, Juhnn YS. cAMP signaling inhibits radiationinduced ATM phosphorylation leading to the augmentation of apoptosis in human lung cancer cells. Mol Cancer 2014; 13: 36.

38 Lee HJ, Cho HS, Jun SY, Lee JJ, Yoon JY, Lee JH et al. Tussilago farfara L. augments TRAIL-induced apoptosis through MKK7/JNK activation by inhibition of MKK7TIPRL in human hepatocellular carcinoma cells. Oncol Rep 2014; 32: 1117-1123.

39 McConnell JL, Gomez RJ, McCorvey LR, Law BK, Wadzinski BE. Identification of a PP2A-interacting protein that functions as a negative regulator of phosphatase activity in the ATM/ATR signaling pathway. Oncogene 2007; 26: 6021-6030.

40 Vannini A, Volpari C, Filocamo G, Casavola EC, Brunetti M, Renzoni D et al. Crystal structure of a eukaryotic zinc-dependent histone deacetylase, human HDAC8, complexed with a hydroxamic acid inhibitor. Proc Natl Acad Sci USA 2004; 101: 15064-15069.

41 Park SY, Jun JA, Jeong KJ, Heo HJ, Sohn JS, Lee HY et al. Histone deacetylases 1, 6 and 8 are critical for invasion in breast cancer. Oncol Rep 2011; 25: 1677-1681.

42 Cole SW, Sood AK. Molecular pathways: beta-adrenergic signaling in cancer. Clin Cancer Res 2012; 18: 1201-1206.

43 Vendetti FP, Rudin CM. Epigenetic therapy in non-small-cell lung cancer: targeting DNA methyltransferases and histone deacetylases. Expert Opin Biol Ther 2013; 13: 1273-1285.

This work is licensed under a Creative Commons Attribution-NonCommercial-ShareAlike

4.0

International License. The images or other third party material in this article are included in the article's Creative Commons license, unless indicated otherwise in the credit line; if the material is not included under the Creative Commons license, users will need to obtain permission from the license holder to reproduce the material. To view a copy of this license, visit http://creativecommons.org/licenses/by-nc-sa/4.0/ 